\title{
San Juan de Dios, la implementación de una cultura de cuidados
}

\section{San Juan de Dios,implementationa culture of care \\ S. João de Deus, a implementação de uma cultura da care}

\begin{abstract}
María de los Santos Oñate Tenorio ${ }^{1}$. Antonio Oñate Tenorio ${ }^{2}$
${ }^{1}$ DUE del Servicio de Nefrología del Hospital Regional Universitario General Carlos Haya de Málaga. Diplomada Universitaria en Podología. Master Universitario en Innovación e Investigación en Cuidados de la Salud: Enfermería Avanzada.

${ }^{2}$ Técnico Superior en Documentación Sanitaria, Administrativo y Celador-Conductor de la Unidad de Atención a la Ciudadanía del Área de Gestión Sanitaria Norte de Cádiz.

Cómo citar este artículo en edición digital: Oñate Tenorio, $M^{a}$ de los S. y Oñate Tenorio, A.. (2013) San Juan de Dios, la implementación de una cultura de cuidados. Cultura de los Cuidados (Edición digital) 17, 36.

Disponible en: http://dx.doi.org/10.7184/cuid.2013.36.05
\end{abstract}

Correspondencia: María de los Santos Oñate Tenorio.Avenida de Europa $n^{\circ} 12^{\circ} \mathrm{H}, 29003$. Málaga.

Teléfonos: 626631706 / 646460335. Coreo electrónico: arcos112@hotmail.com

Recibido 30/01/2013. Aceptado 23/04/2013

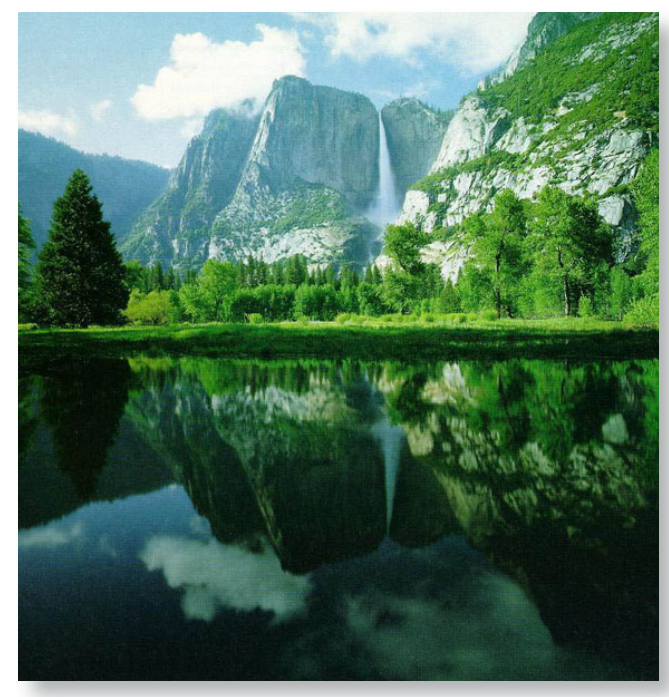

ABSTRACT

Throughout this paper we analyze the influence of St. John of God in healing, because in different and multiple databases appear manifest and publications which mentioned the Holy of St. John of God as a contributor to the care of the sick.

\section{Objective:}

Describe the contributions and impact of San Juan de Dios in the care of the sick, it made for an analytical reflection on them and evaluating whether there is a parallel with the current reality health.

\section{Methodology:}

Performing a literature review through a systematic search of the terms: St. John of God, Care, Culture and Sick in databases: ENFISPO, Dialnet, PubMed, Cochrane Library SciELO and Virtual Andalusian Public Health System, and subsequent reflective analysis of existing documentary research.

\section{Conclusions:}

The contributions of San Juan de Dios involve the implementation of a new culture of care, concerning both organization and to changes in the ways of conceiving the disease, which even today still existing today.

Keywords: San Juan de Dios, Care, Culture, Sick.

\section{RESUMO}

Ao longo deste trabalho, analisar a influência de S. João de Deus na cura, porque em bancos de dados diferentes e múltiplas aparecer manifesto e publicações que 
mencionaram o Santo de São João de Deus, como um contribuinte para o cuidado dos doentes.

\section{Objetivo:}

Descrever as contribuições e impacto de San Juan de Dios no cuidado dos enfermos, feita para uma reflexão analítica sobre eles e avaliar se existe um paralelo com a atual realidade da saúde.

\section{Metodologia:}

Realizar uma revisão bibliográfica através de uma pesquisa sistemática dos termos: São João de Deus, Cuidado, Cultura e Doentes das bases de dados: ENFISPO, Dialnet, PubMed, SciELO e Cochrane Biblioteca Virtual da Andaluzia Sistema Único de Saúde, e posterior análise reflexiva de pesquisa documental existente.

\section{Conclusões:}

As contribuições de San Juan de Dios envolvem a implementação de uma nova cultura de atendimento, tanto em termos de organização e às mudanças nas formas de conceber a doença, que ainda hoje existente hoje.

Palavras-chave: San Juan de Dios, Cuidado, Cultura, Sick.

\section{RESUMEN}

A lo largo de este trabajo analizaremos la influencia de San Juan de Dios en la sanidad, ya que en diferentes y múltiples bases de datos aparecen publicaciones donde se manifiesta y menciona al Santo de San Juan de Dios como un contribuidor de los cuidados a enfermos.

\section{Objetivo:}

Describir las aportaciones y repercusiones de San Juan de Dios en los cuidados de los enfermos, realizando para ello una reflexión analítica sobre los mismos y valorando si existe un paralelismo con la realidad actual sanitaria.

\section{Metodología:}

Realización de una revisión bibliográfica mediante una búsqueda sistemática de los términos: San Juan de Dios, Cuidados, Cultura y Enfermo, en las bases de datos: Enfispo, Dialnet, PubMed, Cuiden, Cochrane Plus, Scielo y Biblioteca Virtual del Sistema Sanitario Público Andaluz, y posterior análisis reflexivo de la investigación documental existente.

\section{Conclusiones:}

Las contribuciones aportadas por San Juan de Dios suponen la implementación de una nueva cultura de cuidados, referentes tanto a organización como a cambios en las formas de concebir la enfermedad, que aún hoy en día en la actualidad siguen vigente.

Palabras clave: San Juan de Dios, Cuidados, Cultura, Enfermo.

\section{INTRODUCCIÓN}

Este es un trabajo analítico que pone de manifiesto la cultura de San Juan de Dios con respecto a las formas de establecer el cuidado de los enfermos.

Independientemente de los aspectos religiosos y del contexto histórico y social que predominan en ésta época, en este trabajo nos centraremos más básicamente en los cuidados que San Juan de Dios prestó a los enfermos en los siglos XV y XVI.

Aunque son muchos los autores y los biógrafos que nos han descrito la figura de San Juan de Dios, en éste estudio nos basaremos en descripción del mismo pero desde una perspectiva sanitaria.

Resalta y predomina de San Juan de Dios el humanismo que transmitía a los enfermos, expresado por nexos de unión entre el enfermo y/o paciente y su cuidador, en una época 
donde la enfermedad era concebida y se entendía como un pecado, hasta tal extremo que cuando una persona enfermaba llamaban al sacerdote y posteriormente y como una tarea subordinada o secundaria llamaban al médico.

San Juan de Dios aportó además grandes reformas en la organización hospitalaria, entre ellas tendremos que resaltar la creación de salas en las que los pacientes eran agrupados no sólo en razón de su sexo sino también por patologías, y en sus escritos se recogen y protocolizan las obligaciones de médicos y enfermeros.

Desde nuestro punto de vista es preciso conocer y analizar a San Juan de Dios ya que aportó una visión humanista al cuidado de los enfermos, aplicando nuevas técnicas y cambios organizativos que contribuyeron a la aparición de una nueva cultura de cuidados a los enfermos y que todavía hoy perdura y permanece en nuestros tiempos siglos después.

Hipótesis de trabajo:

- San Juan de Dios contribuyó a la implementación trascendental de una cultura de cuidados que aportó una nueva forma de ver y tratar al enfermo, realizando una serie de reformas hospitalarias que siguen vigentes en el actual sistema sanitario.

\section{ESTADO DE LA CUESTIÓN}

Juan Ciudad Duarte o Juan de Dios nace al sur de Portugal en Montemayor el Nuevo "Monte Mor o Novo" en 1946 en el seno de una familia humilde.

Con apenas ocho años de edad se traslada a España, su primera infancia discurre en Oropesa (Toledo) donde ejerce el oficio de pastor y otras labores del campo. Posteriormente se dedica a diversos y distintos oficios sin que ninguno de ellos satisfaga sus inquietudes.
A los veintisiete años de edad se alista en el ejército, posteriormente y una vez retirado del mismo se desplaza a Sevilla, Ceuta y después a Granada, donde ejerció trabajos como albañil y librero.

En Granada, un 20 de Enero escucha un sermón del Maestro Juan de Ávila en la Ermita de los Mártires que lo convierte y lo transforma (Larios, 1998), hasta tal punto que es tomado por loco y es ingresado en el Hospital Real de Granada, durante su estancia en éste recibió una serie de cuidados. Es a partir de ese momento y una vez demostrada su cordura, cuando se dedica hasta el resto de sus días al cuidado de enfermos, pobres y más necesitados (González, 2002).

En la prestigiosa Escuela de Medicina y Cirugía y al amparo de los Monjes Jerónimos conoció y aprendió técnicas que posteriormente pondría en marcha, hasta tal punto que abrió su primer hospital y a continuación fundó muchos más, creando una Orden Hospitalaria (Martínez, 2006; Blasco y Espejo, 1995).

San Juan de Dios fue capaz de aportar una visión cristiana al humanismo renacentista (Miralles, 2006), ya que reconoció la grandeza de las personas, y su intención era la de proporcionar y mantener la dignidad de la persona también cuando está enferma.

Fue precisamente en 1535 cuando San Juan de Dios (Martínez, 2006) empieza a ejercer su hospitalidad desde una perspectiva muy distinta a la de la época en que vivía, para él la persona enferma era el elemento clave de todo el sistema sanitario y social, y para atenderla se precisaba de cualidades como capacidad de escucha y dialogo (Morales, 1997), y estar a disposición del enfermo.

Hay autores (Rodríguez, Arribas y Gantes, 2004) que analizan los siguientes valores y principios que definen la cultura institucional 


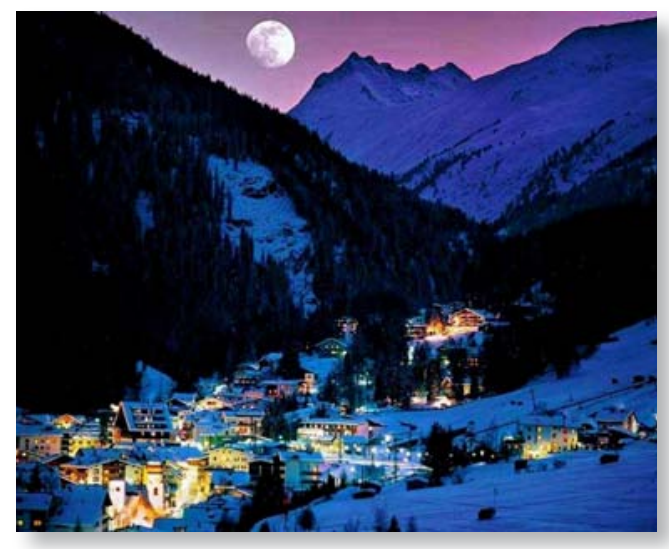

de San Juan de Dios: humanización, hospitalidad, solidaridad, amor, caridad, justicia, libertad, dignidad, comunicación, escucha, considerándolos fundamentales e imprescindibles para el desarrollo de cuidados transculturales y la práctica enfermera. Otros autores reflexionan sobre la construcción social de la historia de la enfermería analizando y describiendo sus ideologías sobre el cuidado (Siles, 2004).

San Juan de Dios fue el fundador de la Orden Hospitalaria de los Hermanos de San Juan de Dios (Benavides, 2010; Miralles, 1995), que aún hoy perdura y sigue en nuestros tiempos. Los principios principales de la misma están basados en el humanismo, considerando al enfermo lo más importante y privilegiado de la Institución, por lo que se le respetará y será tratado con dignidad y con el objetivo final de ofrecerle una asistencia integral mediante la aplicación de todos los avances técnicos ofreciéndole la mejor asistencia técnica, mediante la humanización de sus cuidados, respetando en todo momento los derechos humanos y dándole prioridad a los derechos del enfermo.

Algunos autores han reflexionado sobre el concepto de cuidado y han analizado históricamente el origen fenomenológico de éste (Siles y Solano, 2007). Según Siles (2008) por cuidado se puede entender el producto de las siguientes reflexiones:
- Reflexión teórica: la reflexión sobre las ideas relativas a los cuidados incluye el plano de las creencias y valores que impregnan todo, la atmósfera en la que tienen lugar los cuidados.

- Reflexión práctica: la reflexión sobre los hechos se refiere sobre todo a la valoración de las conductas y eventos observables.

- Y reflexión situacional-contextual-temporal: es la reflexión sobre las circunstancias y se ocupa tanto del marco espacio-temporal como del contexto histórico cultural.

Siles (2001) definió el concepto de cultura como el conjunto de los comportamientos (visibles), pensamientos (ocultos o no), sentimientos (patentes o latentes) y significados (simbólicos) implicados en el proceso de satisfacción de necesidades de un grupo humano. De esta manera, el concepto de cultura no se reduce sólo a los estilos de vida y las conductas evidentes, palpables y fácilmente medibles, sino que es necesario ir a las raíces de esos comportamientos que cuidan en las capas profundas de la sociedad e identificar los factores históricos culturales que determinan los estilos de vida.

Cuando hablamos de la implementación de una cultura de cuidados por parte de San Juan de Dios nos referimos por tanto a la instauración de una concienciación y educación sobre la forma de ejercer y aplicar la asistencia sanitaria al paciente de una forma global y teniendo en cuenta todos los aspectos que pueden influir en el bienestar del mismo.

En este trabajo nos planteamos como objetivo principal conocer la contribución de San Juan de Dios en los cuidados de los enfermos, así mismo también los describiremos y realizaremos una reflexión analítica sobre los mismos, valorando si hay semejanza o correspondencia con los actuales. 


\section{METODOLOGÍA}

La técnica escogida para la realización de este trabajo ha sido la revisión bibliográfica y el análisis de la investigación documental existente. Para ello, y una vez que identificamos las palabras claves y los conceptos fundamentales en DECs y Mesh, se realizó una búsqueda bibliográfica sin límites de fechas de publicación durante los meses de Noviembre y Diciembre de 2012 en las siguientes bases de datos: Enfispo, Dialnet, PubMed, Cuiden, Cochrane Plus, Scielo y Biblioteca Virtual del Sistema Sanitario Público Andaluz.

La estrategia de búsqueda fue la realización de una búsqueda sistemática de estudios realizados y relacionados con San Juan de Dios, siguiendo el mismo método que el recomendado para la búsqueda de otras evidencias clínicas, a través de las distintas bases de datos se seleccionaron artículos en cuyo título o resumen se mencionaba algún dato expreso referente a San Juan de Dios.

\section{RESULTADOS}

Tras realizar un análisis minucioso de toda la información recogida podemos decir que todos los autores coinciden en resaltar la visión humanista y la atención holística que San Juan de Dios aportó al cuidado de los enfermos, haciendo hincapié en los nexos de unión entre paciente y cuidador, reconociendo la dignidad de los enfermos y considerando a éstos como elemento clave (Fernandes de Freitas y Siles, 2008).

Se resalta de San Juan de Dios en la bibliografía revisada la formación que adquirió en nuevas técnicas y habilidades y la creación de protocolos a seguir por el personal sanitario dedicado al cuidado de los enfermos, además la introducción de nuevas reformas en la organización hospitalaria tales como la sectori-

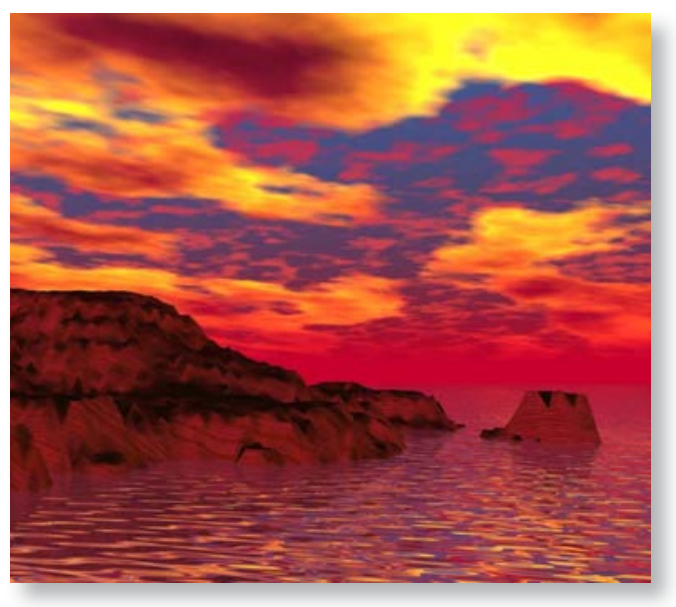

zación de los hospitales por patologías, coincidiendo con otros autores que lo consideran como un reformador (Ventosa, 1995).

Fundó una Orden Hospitalaria y fueron muchos los que siguieron y continuaron con su obra, ofreciendo satisfacción a las necesidades de los pacientes y hospitalidad humanitaria en su asistencia.

\section{DISCUSIÓN}

A lo largo de este trabajo vemos como San Juan de Dios no sólo aportó una visión humanista, sino que cambió el concepto de enfermedad, haciendo que ésta no se considerará como un pecado, sino como se conoce actualmente una alteración estructural o funcional que afecta negativamente al estado de bienestar.

La trascendencia de las aportaciones de San Juan de Dios radica no sólo en la modificación de la cultura de impartir los cuidados a los enfermos, sus formas y la concienciación, proporcionando dignidad y humanización a los cuidados, sino también a nivel organizativo hospitalario, creando en los hospitales una división o sectorización según las patologías, hoy en día podemos comprobar como las especialidades siguen vigente y de hecho todos los hospitales están dividido según este criterio. 
Actualmente los cuidados a los enfermos han adquirido una relevancia muy importante, y para ello se apuesta por la formación de los profesionales y la protocolización de los cuidados, aspectos que San Juan de Dios tuvo muy presentes y que llevó a la práctica.

\section{CONCLUSIONES}

Con esta revisión bibliográfica analítica confirmamos nuestra hipótesis planteada y cumplimos con el objetivo que nos propusimos, ya que este trabajo nos permite conocer las contribuciones aportadas por San Juan de Dios no sólo al cuidado de los enfermos, sino a la sanidad en general.

$\mathrm{Al}$ analizar múltiples fuentes de información hemos llegado a la conclusión de que el trabajo realizado por San Juan de Dios supuso la implementación de una cultura de cuidados, ya que aportó e implementó una nueva forma de ver y de tratar al enfermo, y no sólo eso, también realizo grandes reformas hospitalarias que hoy en día siguen vigentes.

Por tanto, podemos afirmar que existe un paralelismo entre las aportaciones y reformas realizadas por San Juan de Dios en el siglo XVI con el actual sistema sanitario.

\section{BIBLIOGRAFÍA}

- Benavides, F. (2010) El concepto de Patrimonio Cultural en la Orden Hospitalaria de San Juan de Dios. Origen y evolución histórica. Archivo Hospitalario 8, 261-285.

- Blasco, C. y Espejo, D.( 1995). La Orden de San Juan de Dios en Córdoba. Labor asistencial en el hospital de San Lárazo en 1570. Index Enfermería 14, 36-39.

- Fernández, G. y Siles, J. (2008) Antropología y cuidados en el enfoque de San Juan de Dios. Index de Enfermería: información bibliográfica, investigación y humanidades 17, 2, 144-148.

- González, A. (2002) San Juan de Dios, un apóstol a los enfermos y moribundos. Fundación 5, 107-130.
- Larios, J. (1998) San Juan de Dios, de aventurero a santo. Communió: Revista semestral publicada por los Dominicos de la provincia de Andalucía 31, 2, 285-324.

- Martínez, J. (2006) Proceso de beatificación de San Juan de Dios. Biblioteca Autores Cristianos. España.

- Martínez, J.L. (2006) Sobre el nacimiento y procedencia de San Juan de Dios y su obra. Hispania Sacra, Estudios de Edad Moderna 58, 69-100.

- Miralles, MT (1995) San Juan de Dios y su Orden en la Filatelia. Index Enfermería 14, 59-61.

- Miralles, T. (2006) El milagro de San Juan de Dios, de Murillo. Cultura de los Cuidados 20, 50-54.

- Morales, M. L. (1997) Aportaciones psicológicas de Juan Ciudad Duarte (San Juan de Dios). Revista de historia de la psicología 18, 1-2, 227-234.

- Rodríguez, R.M; Arribas, J.M; Gantes, J.C. (2004) Cuatro siglos de cuidados transculturales. Cultura de Cuidados 16, 20-26.

- Siles, J. et al. (2001) Una mirada a la situación científica de dos especialidades esenciales de la enfermería contemporánea: la antropología de los cuidados y la enfermería transcultural. Cultura de los Cuidados 10, 72-87.

- Siles, J. (2004) La construcción social de la historia de la enfermería. Index de enfermería. 13/ 47: 7-10.

- Siles, J. (2008) Historia de la enfermería: una aportación epistemológica desde la perspectiva cultural de los cuidados. Cultura de los Cuidados 24, 5-6.

- Siles, J. y Solano, C. (2007) El origen fenomenológico del cuidado y la importancia del concepto de tiempo en la historia de la enfermería. Cultura de Cuidados 21,1927.

- Ventosa, F. (1995). Juan Ciudad, reflejos de la personalidad humana de un reformador. Index Enfermería 14,15-18. 